\title{
Efecto del nivel del agua sobre la dinámica trófica en un lago de inundación
}

\section{Effect of water level on trophic dynamics in a flood plane lake}

\author{
Manuel Tamara, Alcides Sampedro॰ y Pedro Caraballo®* \\ *Autor de correspondencia: pedro.caraballo@unisucre.edu.co
}

Recibido: 30 octubre de 2018

Aceptado: 02 de abril de 2019

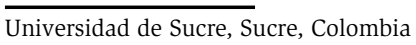

\begin{abstract}
Resumen
Palabras clave: redes tróficas; limnología; detritos; ciénagas

Con el objetivo de definir las interacciones tróficas en el complejo cenagoso de Caimito (municipio de Caimito, departamento de Sucre, $8^{\circ} 47^{\prime} 42,29^{\prime \prime} \mathrm{N}-75^{\circ} 06^{\prime} 48,59^{\prime \prime}$ ) y entre este y los ecosistemas adyacentes, se estudiaron las comunidades biológicas presentes. Se utilizó la información obtenida en estudios previos, dos en períodos de aguas altas y uno en período de aguas bajas, en cada uno de ellos, se estudió la riqueza biótica del cuerpo de agua y el ambiente aledaño, los grupos bióticos estudiados fueron: aves, macrófitas acuáticas, macroinvertebrados acuáticos, peces, zooplancton y reptiles. Se hizo una lista de las especies y de grupos taxonómicos hallados y se establecieron trofoespecies, con las que se realizó una matriz binaria de datos, que sirvió de base para construir la red trófica. Se evidenció el papel importante del detritus, como una fuente de energía en este tipo de ecosistemas. Los grupos más representativos fueron los peces omnívoros y el conjunto de macroinvertebrados acuáticos, cuyo amplio espectro trófico y mecanismos de obtención del alimento, los convierte en grupos vitales en el aprovechamiento de materia y energía y transporte a los niveles tróficos superiores. Las redes tróficas que representan los períodos de sequía e inundación son funcional y estructuralmente diferentes. En el período de sequía, la comunidad es dependiente del detritus y en la inundación participan los productores primarios.
\end{abstract}

\begin{abstract}
Biological communities were studied in order to define the trophic interactions in the Caimito swamp complex (municipality of Caimito, department of Sucre, $84^{\circ} 7^{\prime} 42.29^{\prime \prime} \mathrm{N}-75^{\circ} 06^{\prime} 48.59^{\prime \prime} \mathrm{O}$ ), and between this swamp complex and adjacent ecosystems. Information obtained in previous studies was used, one of them carried out in the high water period and another one in the low water period, as well as a sampling done by the authors during the high water period. In each of them, the biotic richness of the body of water and the surrounding environment was studied: the biotic groups were: birds, aquatic macrophytes, aquatic macro invertebrates, fish, zooplankton and reptiles. A list was made of the species and taxonomic groups found, and trophic species were established, which served as the basis for the construction of the food web. The important role of detritus as a source of energy was evidenced in this ecosystem. Most representative groups were the omnivorous fish and the aquatic macro invertebrates. Their broad trophic spectrum and mechanisms for obtaining food make them vital groups in terms of the use of matter and energy, and transport to higher trophic levels. The food webs that represent the periods of drought and flood are functionally and structurally different. In the drought period, the community depends on detritus, and primary producers participate during.
\end{abstract}

Key words: 


\section{Introducción}

Los lagos de inundación, localmente conocidos como ciénagas, son cuerpos de agua asociados directa 0 indirectamente con un río, y su dinámica biológica e hidráulica depende del pulso anual de inundación del río (Junk et al., 1989). Esta conexión genera en los lagos de inundación cuatro períodos limnológicos diferentes entre sí (seca, inundación, llena y bajante) descritos con detalle por Rai y Hill (1984). Estos períodos, son eventos de gran magnitud en los que incluso la contribución relativa de materia orgánica autóctona y alóctona puede cambiar (Caraballo et al., 2012; 2014). Esta variabilidad ambiental, ocasionada por el pulso de inundación produce variaciones, entre otras, en las dinámicas poblacionales (Bayley et al., 2018) y en los procesos reproductivos (Callil et al., 2018). Por ejemplo, en la ciénaga de Ayapel, asociada al río San Jorge al igual que la de Caimito, la dinámica hidrológica y la conectividad al río (Ochoa-Orrego et al., 2015), determinan el ingreso o salida de peces y les provee de zonas seguras de desove y desarrollo. En general es de esperarse que la interrelación entre la estructura de la comunidad ecológica, la estabilidad y los procesos que ocurren dentro del ecosistema que constituyen la base de las relaciones tróficas, también cambien en esos períodos.

De acuerdo con Schindler y Scheuerell (2002), existe un vacío acerca de las causas y consecuencias del acoplamiento ecológico entre los hábitats pelágicos, bentónicos y ribereños. Este vacío puede ser abordado mediante el análisis de las relaciones tróficas, definidas por los recursos alimenticios y la depredación, que son algunos de los factores limitantes del crecimiento poblacional de cualquier especie por efecto del control ascendente y descendente (Lynam et al., 2017). Así, las interacciones tróficas son componentes esenciales para entender la dinámica de las comunidades $\mathrm{y}$, consecuentemente los modelos emergentes de coexistencia y diversidad en los ecosistemas (Giacomini y Petrere, 2010).

Se trata de la forma más elemental y efectiva de visualizar una comunidad, ya que las interacciones alimenticias permiten mapear el flujo de materiales y nutrientes, identificando las relaciones tróficas importantes a fin de definir una estructura que pueda ser comparada con otros ecosistemas (Jepsen y Winemiller, 2002; Vanni, 2002), porque son representaciones gráficas de las relaciones que existen entre predadores y presas de una comunidad.
Entre los recursos alimenticios de un ecosistema, los productores primarios ocupan un lugar muy importante. En general, para los lagos de inundación se han definido cuatro grandes grupos de productores primarios: las macrófitas acuáticas, la floresta inundable, el fitoplancton y el perifiton (Araujo-Lima et al., 1986); a partir de los cuales se genera gran parte del carbono que sustenta la producción pesquera. A lo anterior se agrega el detritus, que se incluye como una trofoespecie especial por su aporte energético (Pimm et al., 1991), su influencia en la composición de la red alimentaria y dinámica, aumentando la estabilidad y la persistencia del sistema, y los efectos sustanciales que tiene sobre la estructura trófica y la diversidad (Moore et al., 2004).

El análisis de las redes tróficas se desarrolla con base en el número de especies (o trofoespecies), la densidad de uniones, que expresa la estabilidad de la red (Dunne et al., 2002) y la conectancia, que define la proporción de conexiones existentes, en función del número total de conexiones posibles (Pimm et al., 1991). Por su parte, Bersier et al. (2002) proponen un análisis cuantitativo, relacionado con el grado de centralidad el cual se refiere al número de contactos directos que tiene una especie en la red, divididos en grados de entrada y grados de salida que dependen de la dirección del flujo.

Otro tipo de análisis que puede hacerse es simular diversas situaciones o escenarios que alteren la disposición y el número de trofoespecies en la red, lo que permite explorar lo que pasaría en un determinado ecosistema ante distintos escenarios ambientales. Por ello se planteó como objetivo comparar las estructuras tróficas de la Ciénaga de Caimito en épocas de aguas altas y bajas, considerando la enorme fluctuación que se presenta entre estos períodos limnológicos contrastantes.

\section{Materiales y métodos}

\section{Área de estudio}

El municipio de Caimito se encuentra ubicado al sur del departamento de Sucre, Colombia (figura 1). Presenta dos períodos climáticos marcados: pluviosidad alta, que va desde abril hasta diciembre, y pluviosidad baja, que comprende los meses de diciembre a abril. La temperatura promedio de la región es de $28{ }^{\circ} \mathrm{C}$ (www.clima.com). Este territorio se halla en la cuenca del río San Jorge, y su casco urbano limita con un complejo cenagoso conformado por tres ciénagas: Zarzal, Hoja Ancha y la Ciénaga de Caimito (IGAC, 1991) (figura 1). 

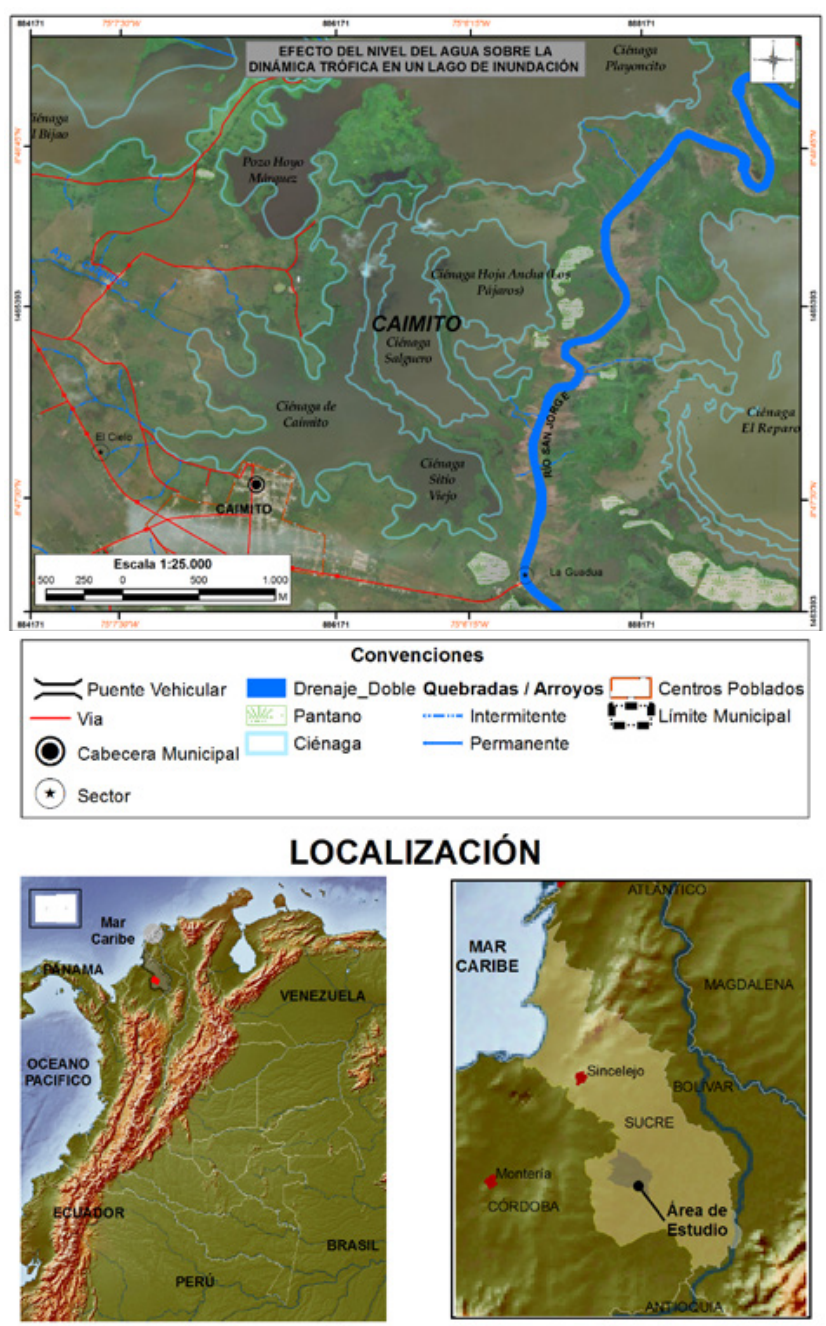

Figura 1. Ubicación geográfica de Caimito y del complejo cenagoso de Caimito. (Imagen tomada y modificada de: Google Earth).

\section{Fase de campo}

Se realizaron dos recorridos de dos días: uno en período de aguas bajas, en octubre de 2014 y uno en aguas altas, en abril de 2015. En ambos se enlistaron distintos grupos de organismos que habitan la Ciénaga de Caimito: aves, zooplancton, peces, macroinvertebrados acuáticos, reptiles, macrófitas acuáticas, vegetación ribereña y adyacente. En un segundo momento, se hizo uso de información secundaria para complementar los datos obtenidos. Con ello se consiguió añadir los mamíferos voladores y no voladores al inventario previamente establecido, así como saber el hábito alimenticio de cada taxón. Para ello se recurrió a los trabajos de Lamadrid y Paternina (2003),
Geney y Benedetti (2003) y Durán y Canchila-Pérez (2015). Para la definición de las trofoespecies, se hicieron las agrupaciones basadas en la similitud de presas y depredadores dentro del ecosistema (Pimm et al., 1991), por lo que se le otorgó más relevancia a la función dentro del ecosistema de un determinado organismo que a su posición taxonómica. Los datos de los dos muestreos se unieron para obtener la mejor visión posible de los grupos tróficos presentes en la ciénaga.

Después de definir el grupo de trofoespecies, se realizó una matriz binaria de datos (1: relación trófica, 0 : no relación), la cual fue ingresada en el software Gephi 0.9.1 donde se generó la red trófica gráfica. En ella se evidenciaron de forma más clara, las interacciones entre los organismos. Luego se analizó la red construida en términos de su topología, conectancia y densidad de uniones (Pimm et al., 1991; Dunne, 2009), así como descriptores cuantitativos propuestos por Bersier et al. (2002).

Por último, se hicieron simulaciones en el software Gephi 0.9.1 de diferentes escenarios posibles donde se recrearon el pulso de inundación del río y la desaparición de distintos grupos de productores (desde el período de inundación, donde se contó con la totalidad de las trofoespecies definidas). Luego se llevó acabo, la primera simulación en descenso de aguas, que ocasiona la desconexión del cuerpo de agua con los cultivos y vegetación ribereña establecidos a su alrededor y en la zona de transición acuático terrestre (ATTZ, por sus siglas en inglés) y demás plantas terrestres ubicadas en las cercanías, la salida de las macrófitas flotantes del río y de las macrófitas enraizadas a la ATTZ por la pérdida de agua de la ciénaga. La ausencia de macrófitas conllevaría a la escasez del perifiton, por lo que, en la segunda simulación, este fue retirado de la red. En la tercera se eliminó el fitoplancton, debido a que la transparencia disminuye (Hernández-Atilano et al., 2008) y con ello, la incidencia de luz, dejando solamente al detritus como fuente primaria de energía en el ecosistema. Al final se analizaron y compararon los descriptores ya mencionados.

\section{Resultados}

En período de aguas altas, los hábitos alimenticios fueron distribuidos en distintos niveles tróficos, entre los que se hallaron: productores (detritus, perifiton, fitoplancton, macrófitas acuáticas, vegetación riparia, cultivos, árboles frutales y de fruto seco), detritívoros (macroinvertebrados acuáticos raspadores, colectores-raspadores, raspadores y trituradores como Chironomidae), filtradores (peces 
filtradores), herbívoros (peces herbívoros, aves herbívoras, reptiles herbívoros e insectos herbívoros), carnívoros (aves y reptiles carnívoros, zooplancton carnívoro), granívoros (aves granívoras), piscívoros (peces piscívoros, aves piscívoras, murciélagos piscívoros, mamíferos piscívoros, reptiles piscívoros), insectívoros (peces insectívoros, aves insectívoras y reptiles insectívoros), omnívoros (peces omnívoros, aves omnívoras, mamíferos omnívoros, reptiles omnívoros Macrobrachium sp.) y nectarívoros (insectos polinizadores) (figura 2).

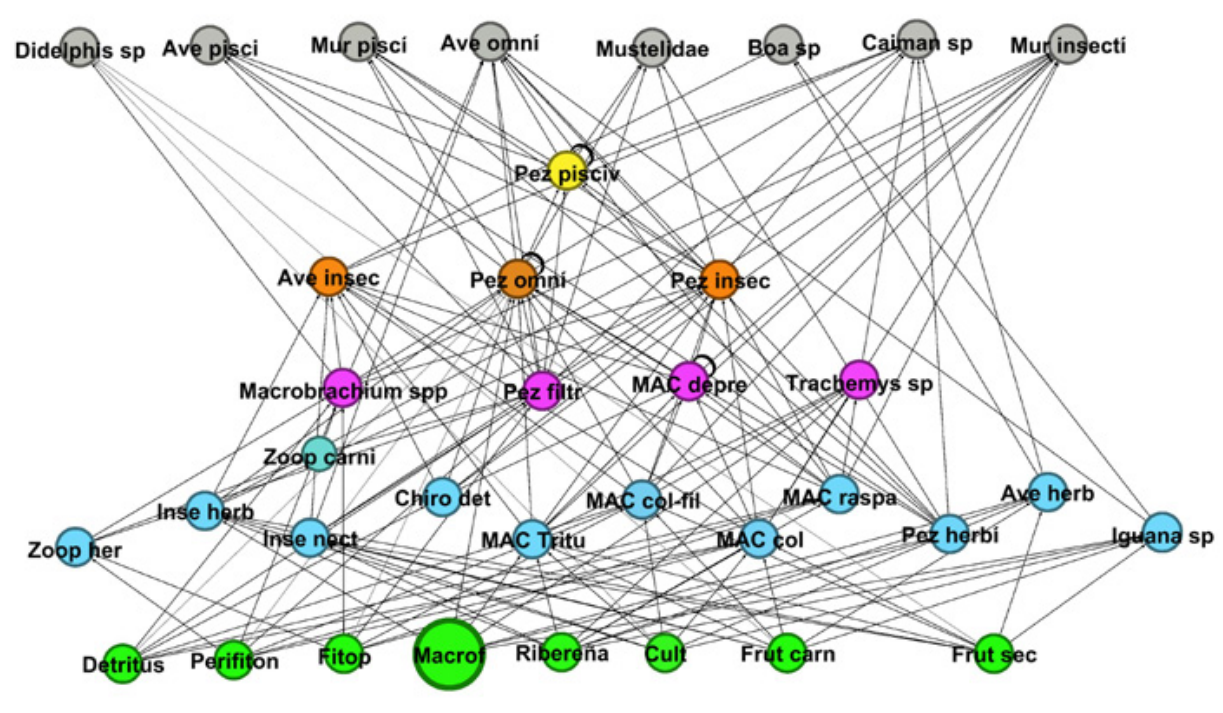

Figura 2: Red trófica de la Ciénaga de Caimito en periodo de aguas altas con todas las fuentes de producción primaria disponibles para el sistema.

Se conformaron 14 categorías tróficas, distribuidas en 36 trofoespecies, 166 conexiones nueve niveles tróficos, máximo densidad de uniones de 4,6 y conectancia interespecifica de 0,12 . Las trofoespecies y sus proporciones se distribuyeron de la siguiente manera: ocho son de tope, lo que equivale al 22,2 \%, 20 intermedias $(55,5 \%)$ y ocho basales, que serían el 22,2 $\%$ de los nodos. Las mediciones de estos parámetros y de los índices cuantitativos producto de las simulaciones se encuentran en la tabla 1.

Tabla 1. Parámetros medidos en las simulaciones hechas para observar el efecto de condiciones ambientales adversas sobre la red.

\begin{tabular}{lrrrr}
\hline & Trofoespecies & Conexiones & Densidad & Conectancia \\
\hline Aguas altas & 36 & 166 & 4,6 & 0,12 \\
Salida de aguas & 25 & 97 & 3,8 & 0,15 \\
Sequía & 23 & 82 & 3,5 & 0,15 \\
Extrema sequía & 20 & 72 & 3,6 & 0,18 \\
\hline
\end{tabular}

En período de aguas altas, resaltan los peces omnívoros, debido a que tienen 10 conexiones de entrada (presas) y 6 de salida (predadores). También se observa que los macroinvertebrados acuáticos transportan gran cantidad de materia y energía hacia los niveles tróficos superiores, sumando el conjunto de interacciones de estos grupos y no 27 por individual.
En la primera simulación (figura 3), donde se propone la ausencia de las macrófitas acuáticas y la separación de la ciénaga del medio terrestre, se observó que desaparecieron 15 trofoespecies, el valor de la densidad de uniones disminuyó y la conectancia aumentó. Los organismos filtradores, detritívoros colectores y raspadores se convirtieron en los únicos encargados de ser los puentes entre el nivel trófico basal y los superiores. 


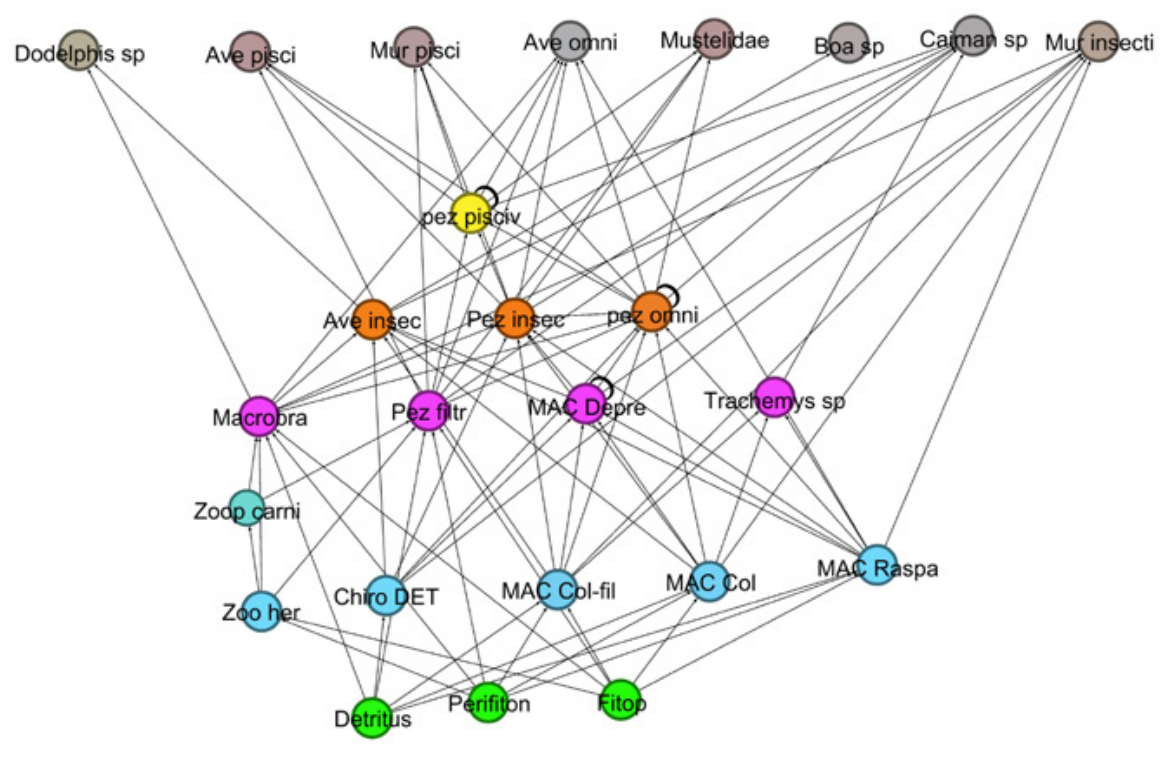

Figura 3. Red trófica de la Ciénaga de Caimito, al inicio de la sequía, sin las macrófitas acuáticas, vegetación riparia y árboles maderables.

En el segundo supuesto (figura 4), la falta del perifiton conlleva a un efecto de extinción en cascada mínimo, en el que desaparece solo una trofoespecie. Sin embargo, la densidad de uniones se mantuvo a la baja, pero la conectancia se mantuvo casi en el mismo valor que en el supuesto anterior. En la última situación simulada (figura 5), siendo el detritus la única fuente de producción primaria, el número de niveles tróficos disminuyó a ocho, los peces filtradores se convirtieron en los principales transportadores de nutrientes, debido a que presentaron siete depredadores dentro de la red, y la densidad de uniones se comportó distinto a las simulaciones anteriores, subiendo levemente, igual que la conectividad.

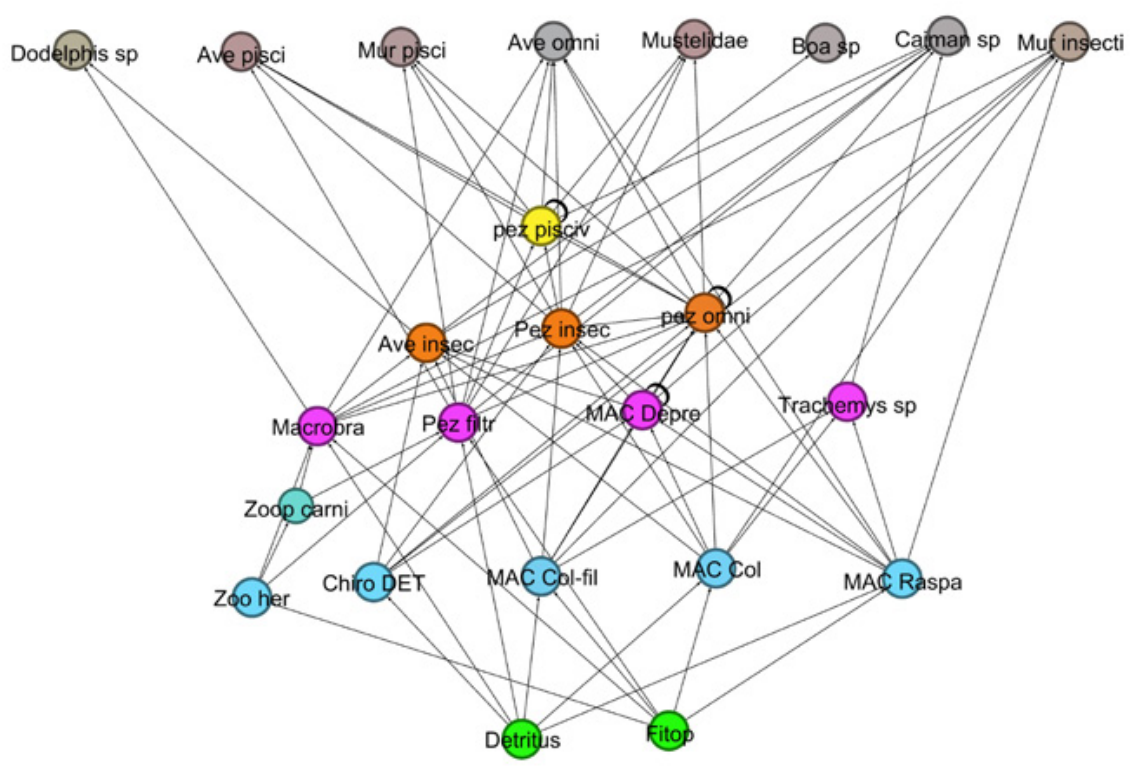

Figura 4. Red trófica de la Ciénaga de Caimito en condición de aguas bajas, sólo con el fitoplancton y el detritus como fuentes de energía y los efectos que esto provoca sobe la red. 


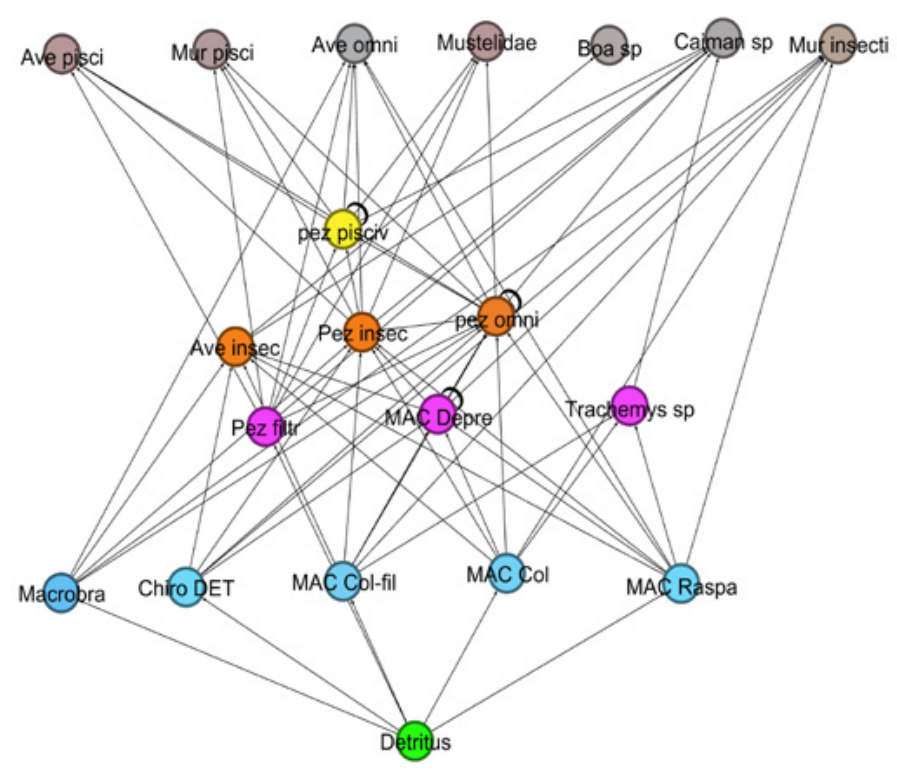

Figura 5. Red trófica de la Ciénaga de Caimito, en las condiciones ambientales de sequía extrema, baja profundidad y mucha turbidez. La red se hace totalmente dependiente del detritus y la fragilidad de las conexiones aparece mayor.

\section{Discusión}

Un modo de hacer una comparación clara referente a la estabilidad de una red trófica respecto a otras, es mediante el uso de parámetros y descriptores topológicos. Otra, es analizándolas funcionalmente e identificando uno o varios grupos de importancia en el funcionamiento de la misma, teniendo siempre en cuenta la influencia del nivel del agua de la ciénaga, definida fuertemente por el pulso de inundación (Junk et al., 1989).

El número de trofoespecies definidas en una red trófica, está ligado al nivel de resolución en la agrupación de organismos decidido por el investigador. En este estudio se establecieron 39 trofoespecies, número que contrasta con un estudio enfocado en las relaciones entre peces y macroinvertebrados (López van Oosterom et al., 2013), donde se definieron 23 trofoespecies, lo que sugiere que el número de grupos definidos podría estar influenciado también por los taxones específicos con los que se decida trabajar (Andramunio-Acero y Caraballo, 2012).

Las proporciones entre trofoespecies basales, intermedias y de tope en la red trófica en periodo de aguas altas, es congruente con la descripción de Bersier et al. (2002), quienes indicaron que generalmente, la suma de las proporciones entre presas y consumidores (N:P) se agrupan en valor cercano a uno. En este caso fue igual a uno, patrón que parece ser inevitable en las redes grandes (Closs et al., 1993) con mayor proporción de trofoespecies intermedias (Martínez y Lawton, 1995). En la práctica, este valor sugiere que todas las trofoespecies productoras tienen la misma cantidad de presas que los consumidores. Esta proporción también es descrita como constante en las redes tróficas, indistintamente del número de trofoespecies y del hábitat (Pimm, 2002), y es importante en el flujo de energía, ya que se relaciona con una elevada redundancia funcional, por lo que, en caso de pérdida de algunos nodos, el flujo de materia y energía hacia los niveles superiores de la red, está asegurado (Covich et al., 1999), tal como se observó en las simulaciones.

En período de aguas altas (figura 2 y tabla 1), el valor de la densidad de uniones de 4,6 indica que, en promedio, las trofoespecies están relacionadas con 4,6 trofoespecies, distribuidas igualmente entre presas y depredadores (Bersier et al., 2002); sin embargo, otros autores han hallado que la proporción de presas es mayor a la proporción de predadores de una trofoespecie (Lindeman, 1942; Odum, 1971; Menzie, 1980), como se observó en este estudio. Esta característica puede ser importante en la dinámica trófica del ecosistema debido a la pérdida de materia y energía de un nivel a otro, por lo que tener el mismo número de relaciones de presadepredador podría acarrear un flujo menos eficiente y una red más corta y menos estable. 
La densidad de uniones disminuyó progresivamente mientras se retiraban trofoespecies de la red, pasando de 4,6 en inundaciones a 3,6 en la sequía (tabla 1), esto puede deberse a que los grupos retirados contribuyeron ampliamente en la producción de energía en la comunidad, y su ausencia produce a una disminución en la interrelación de los grupos tróficos. El valor de 0,12 en la conectancia, en el período de inundación, sugiere estabilidad en la red (Dunne, 2009), distinto a lo que se va observando en las simulaciones (tabla 1), donde llega a 0,18 y la hace inestable. Estos valores contrastaron con el de otros estudios, debido a que es común que al aumentar el número de trofoespecies, disminuya el valor de la conectividad. Por otro lado, Pimm et al. (1991) sugieren que incluir al detritus como trofoespecie funcional, ocasionará un aumento de la conectividad. Esto es más evidente cuando, como en este estudio, se incluyen las relaciones tróficas con organismos externos al cuerpo de agua. Además, la naturaleza de las trofoespecies, así como su alto o bajo grado de relaciones alimenticias, puede jugar un papel importante a la hora de estimar estos parámetros.

El porcentaje de omnívora encontrado fue de 53,5 \% de nodos consumidores, lo que puede ser una respuesta de los organismos a las condiciones fluctuantes de sus hábitats (Jepsen y Winemiller, 2002). Uno de los grupos más representativos son los peces omnívoros y los macroinvertebrados acuáticos, cuyo amplio espectro alimenticio y modos de obtención de alimento, los lleva a consumir organismos en todos los niveles tróficos inferiores a los suyos (Andramunio-Acero y Caraballo, 2012; Atencia- Gandra et al., 2017). Esta cualidad hace que los pasos entre un nivel trófico y otro, sean menores a los que serían si el transporte de materia y energía se diera pasando por todos los niveles de la red, lo que en última instancia significaría un transporte más eficiente de recursos que se perderían por la llamada espina de Lindeman” (Lindeman, 1942).

El detritus, resalta su importancia en período de sequía, debido a que en lagos de inundación puede tener fuertes influencias en la estructura y dinámica de la red (Polis et al., 1997), suministrando energía que puede sostener mayores densidades de consumidores de lo que se mantendría si estos consumidores se alimentaran exclusivamente con energía producida por el fitoplancton. De este modo los detritívoros se mantienen en la red mediante el consumo de detritus de origen alóctono y autóctono (Schaus y Vanni, 2000; Vanni, 2002).
Las redes alimenticias varían en la medida en que el detritus se deriva de fuentes alóctonas o autóctonas y la forma particular en que se producen. Para integrar el detritus en la comprensión de los factores que impulsan la función y la diversidad de organismos, poblaciones y ecosistemas, se deben reconocer los vínculos íntimos entre detritus y otros componentes de los sistemas vivos, así como la heterogeneidad y ontogenia de los recursos detríticos (Moore et al., 2004).

Para este estudio, se consideran al conjunto de macroinvertebrados acuáticos y a los peces omnívoros, como trofoespecies clave. Esta postura es nueva respecto a la tendencia de siempre nombrar un solo grupo, por lo que esta posición deberá ser evaluada a futuro con estudios dirigidos al flujo especifico de energía de dichas trofoespecies y cuantificar su importancia dentro del sistema. El número de trofoespecies tope encontradas y persistentes a lo largo de los periodos hidrológicos simulados, indican que el ecosistema exporta gran cantidad de energía, lo que es coherente con la dinámica expresada por el pulso de inundación.

La vegetación ribereña, las aves acuáticas, los insectos emergentes, entre otros, sirven como rutas bidireccionales de materia y energía entre los ecosistemas acuático y terrestre. La reducción del tamaño de la ciénaga, hace que aumente la intensidad y frecuencia de las interacciones tróficas, fenómeno que quedó evidenciado al mostrar el aumento de la conectancia, luego de haber realizado las simulaciones.

\section{Referencias}

Andramunio-Acero, C. P. y Caraballo, P. 2012. Análisis de las relaciones tróficas en un lago de inundación de la Amazonia colombiana. Revista Colombiana de Ciencia Animal-RECIA 4(1): 102-120.

Araujo-Lima, C., Forsberg, B., Victoria, R. y Martinelli, L. 1986. Energy sources for detritivorous fishes in the Amazon. Science 234(4781): 1256-1258.

Atencia Gándara, P., Conde, Ó. y Solano, L. 2017. Uso de recursos biológicos por Elachistocleis pearsei (Ruthven, 1914) (Anura: Microhylidae) en un ecosistema léntico del departamento de Sucre, Colombia. Acta zoológica mexicana 33(1): 130-132.

Bayley, P., Castello, L., Batista, V. y Fabré, N. 2018. Response of Prochilodus nigricans to flood pulse variation in the central Amazon. Royal Society Open Science 5(6): 1-15. 
Bersier, L.F., Banašek-Richter, C. y Cattin, M.F. 2002. Quantitative descriptors of food-web matrices. Ecology 83(9): 2394-2407.

Callil, C., Leite, M., Mateus, L. y Jones, J. 2018. Influence of the flood pulse on reproduction and growth of Anodontites trapesialis (Lamarck, 1819) (Bivalvia: Mycetopodidae) in the Pantanal wetland, Brazil. Hydrobiologia 810(1): 433-448.

Caraballo, P., Fosberg, B. y Leite, R. 2012. Papel trófico del microbial loop en un lago de inundación en la Amazonía Central. Acta Biológica Colombiana 17(1): 103-116.

Caraballo, P., Forsberg, B. y Leite, R. 2014. Seasonal variation in the distribution and isotopic composition of phytoplankton in an Amazon floodplain lake, Brazil. Acta Biológica Colombiana 19(2): 291-304.

Closs, G., Watterson, G.A. y Donnelly, P.J. 1993. Constant Predator-Prey Ratios: An Arithmetical Artifact?. Ecology 74(1): 238-243.

Covich, A, Palmer, M. y Crowl T. 1999. The role of benthic invertebrate species in freshwater ecosystems: zoobenthic species influence energy flows and nutrient cycling. BioScience 49(2): 119-127.

Dunne, J, Williams, R., y Martínez, N. 2002. Food-web structure and network theory: the role of connectance and size. Proceedings of the National Academy of Sciences 99(20): 12917-12922.

Dunne, J. 2009. Food webs. En: Meyers, R.A., Editor. Encyclopedia of complexity and systems science. Springer, Nueva York.

Durán, A., y Canchila-Pérez, S. 2015. Ensamblaje de murciélagos (Mammalia: Chiroptera) en dos zonas del departamento de Sucre, Colombia. Acta zoológica mexicana (31)3: 358-36.

Geney, G. y Benedetti, C. 2003. Análisis de la extracción pesquera artesanal en el complejo cenagoso del municipio de Caimito (Sucre) durante la época de aguas altas. Trabajo de pregrado, Universidad de Sucre, Sucre, Colombia.

Giacomini, H. y Petrere, M. 2010. A estrutura de teias tróficas. Boletim da Sociedade Brasileira de Limnologia 38(1): 1-33.

Hernández-Atilano, E., Aguirre, N., Palacio, J. y Ramírez, J. 2008. Variación espacio temporal de la asociación fitoplanctónica en diferentes momentos del pulso hidrológico en la ciénaga de Ayapel (Córdoba), Colombia. Actualidades Biológicas 30(88): 67-81.

Instituto Geográfico Agustín Codazzi (IGAC). 1991. Carta Catastral. Instituto Geográfico Agustín Codazzi.

Jepsen, D.B. y Winemiller, K.O. 2002. Structure of tropical river food webs revealed by stable isotope ratios. Oikos 96: 46-55.

Junk, W., Bayley, P.B. y Sparks, R.E. 1989. The flood pulse concept in river-floodplain systems. En: Dodge, D.P., Editor. Proceedings of the International Large River Symposium (LARS). Canadian Special Publication of Fisheries and Aquatic Sciences, Ottawa - Ontario.

Lamadrid, P. y Paternina, A. 2003. Macroinvertebrados bentónicos y su relación con el "pulso" de inundación del río San Jorge en el complejo lagunar de Caimito (Sucre). Trabajo de pregrado, Universidad del Atlántico, Atlántico.

Lindeman, R. 1942. The trophic-dynamic aspect of ecology. Ecology 23(4): 399-417.

López van Oosterom, M.V, Ocón, C.S, Brancolini, F., Maroñas, M.E, Sendra E. D, Rodríguez Capítulo, A. 2013. Trophic relationships between macroinvertebrates and fish in a pampean lowland stream (Argentina). Iheringia. Série Zoologia 103(1): 57-65.

Lynam, C., Llope, M., Möllmann, C., Helaouët, P., BaylissBrown, G. y Stenseth, N. 2017. Interaction between top-down and bottom-up control in marine food webs. Proceedings of the National Academy of Sciences 114(8): 1952-1957.

Martínez, N., y Lawton, J. 1995. Scale and food-web structure - from local to global. Oikos 73(2): 148-154.

Menzie, C.A. 1980. Potential significance of insects in the removal of contaminants from aquatic systems. Water, Air, and Soil Pollution 13(4): 473-479.

Moore, J., Berlow, E., Coleman, D., Ruiter, P. y Dong, Q., Hastings, A. y Nadelhoffer, K. 2004. Detritus, trophic dynamics and biodiversity. Ecology letters 7(7): 584-600.

Ochoa-Orrego, L., Jiménez, L. y Palacio, J. 2015. Ictioplancton en la ciénaga de Ayapel, río San Jorge (Colombia): cambios espacio-tempo. Boletín Científico. Centro de Museos. Museo de Historia Natural 19(1): 103-114.

Odum, E. 1971 Fundamentals of ecology. Saunders, Philadelphia, Pennsylvania. 
Pimm, S. 2002. Food Webs. The University of Chicago Press, Londres.

Pimm, S., Lawton, J. y Cohen, J. 1991. Food web patterns and their consequences. Nature 350: 669-674.

Polis, G., Anderson, W. y Holt, R. 1997. Towards an integration of landscape and food web ecology: the dynamics of spatially subsidized food webs. Ecology System 28: 289-316.

Rai, H. y Hill, G. 1984. Primary production in the Amazonian aquatic ecosystem. In The Amazon, Springer, Dordrecht.
Schaus, M. y Vanni, M. 2000. Effects of gizzard shad on phytoplankton and nutrient dynamics: Role of sediment feeding and fish size. Ecology 81: 1701-1719.

Schindler, D.E. y Scheuerell, M.D. 2002. Habitat coupling in lake ecosystems. Oikos 98(2): 177-189.

Vanni, M. 2002. Nutrient cycling by animals in freshwater ecosystems. Ecology System 33: 341-370

Citar como: Tamara, M., Sampedro, A. y Caraballo, P. 2019. Efecto del nivel del agua sobre la dinámica trófica en un lago de inundación.

Intropica 14(1): 24-32. DOI: http://dx.doi.org/10.21676/23897864.2871 